size of the eliminants but the size of their largest prime factor which is important, and secondly it is not essential to take the $m$ 's in order of magnitude. In answer, it should be pointed out that after one passes the limits of factor tables, it becomes impracticable to deal with the factors of the eliminant rather than the eliminant. Therefore, since the eliminant (in one case at least) appears to be an increasing function of $m$, one is compelled to work with monotone increasing $m$.

Cornell University

\title{
SOME UNIFORMLY CONVEX SPACES
}

\section{R. P. BOAS, JR. ${ }^{1}$}

1. Introduction. A Banach space is said to be uniformly convex if to every $\epsilon, 0<\epsilon<1$, there is a $\delta(\epsilon), 0<\delta(\epsilon)<2$, such that $\|x\|=\|y\|=1$ and $\|x-y\| \geqq \epsilon$ imply $\|x+y\|<2-\delta(\epsilon)$. J. A. Clarkson, who introduced the concept of uniform convexity [5], proved that the spaces $L^{p}$ and $l^{p}$ are uniformly convex if $p>1$, basing his proof on the following inequalities ${ }^{2}$ among norms of elements of $L^{p}$ or $l^{p}$ :

$$
\begin{array}{llr}
\|x+y\|^{p}+\|x-y\|^{p} \leqq 2^{p-1}\left(\|x\|^{p}+\|y\|^{p}\right), & p \geqq 2 ; \\
\|x+y\|^{p}+\|x-y\|^{p} \leqq 2\left(\|x\|\left\|^{p^{\prime}}+\right\| y\|\|^{p^{\prime}}\right)^{p-1}, & p \geqq 2 ; \\
\|x+y\|^{p^{\prime}}+\|x-y\| \|^{p^{\prime}} \leqq 2\left(\|x\|\left\|^{p}+\right\| y\|\|^{p}\right)^{p^{\prime}-1}, & 1<p \leqq 2 .
\end{array}
$$

The uniform convexity of $L^{p}$ and $l^{p}$ follows by inspection from either (1.1) or (1.2) if $p \geqq 2$, and from (1.3) if $1<p \leqq 2$. As Clarkson observed, (1.1) is a consequence of (1.2), since $\left\{(1 / 2)\left(a^{r}+b^{r}\right)\right\}^{1 / r}$ is an increasing function of $r$ for positive $a$ and $b[6$, p. 26], so that the right side of (1.1) is not less than that of (1.2). However, (1.1) is interesting because it is considerably simpler to prove than (1.2) (see $\S 3$ ), so that the uniform convexity of $L^{p}$ and $l^{p}$ can be established more easily for $p \geqq 2$ than for $1<p<2$.

In this note I give a short proof of Clarkson's inequalities (and of a general set of inequalities, which includes them), using M. Riesz's convexity theorem for linear forms. This proof has the advantage that it can be generalized to show that the spaces $L^{p}\left\{L^{q}\right\}, L^{p}\left\{l^{q}\right\}$, $l^{p}\left\{L^{q}\right\}, l^{p}\left\{l^{q}\right\}$ are all uniformly convex ${ }^{3}$ if $p>1, q>1$. Here $L^{p}\{E\}$ is

1 National Research Fellow.

2 Here, as throughout this note, $p^{\prime}=p /(p-1)$; similarly for other letters.

${ }^{3}$ These results suggest the possibility that $L^{p}\{E\}$ and $l^{p}\{E\}$ are uniformly convex whenever $E$ is; but I can offer no evidence for or against this conjecture. 
the Banach space of functions $x(t)$ from a real interval to the Banach space $E$, integrable (in the sense of Bochner [3]), and such that $\mathfrak{M}_{p}[x]=\left(\int\|x(t)\|{ }^{p} d t\right)^{1 / p}$ is finite; the norm of $x$ in $L^{p}\{E\}$ is $^{4} \mathfrak{M}_{p}[x]$. Similarly, $l^{p}\{E\}$ is the Banach space of sequences $X=\left\{x_{n}\right\}$ of elements of $E$, such that $\mathfrak{M}_{p}[X]=\left(\sum\left\|x_{n}\right\|^{p}\right)^{1 / p}$ is finite, with $\mathfrak{M}_{p}[X]$ as the norm of $X$.

I also give a separate proof of (1.1); this proof is strictly elementary, depending only on the inequalities of Hölder, Minkowski, and Jensen.

Clarkson's inequalities can be written in the general form

$$
(\|x+y\| r+\|x-y\| r)^{1 / r} \leqq 2^{1 / s^{\prime}}\left(\|x\|^{s}+\|y\|^{s}\right)^{1 / s}
$$

(1.4) reduces to (1.1), (1.2), (1.3) for $r=s=p ; r=p, s=p^{\prime} ; r=p^{\prime}$, $s=p$. In each case, $s \leqq p \leqq r$; this condition will be used in $\$ 2$.

2. A preliminary reduction. We begin by showing that (1.4), for an $r$ and an $s$ with $1<s \leqq p \leqq r$, is implied by the same inequality for complex $x$ and $y$ (with norms replaced by absolute values). In fact, if we know

$$
(|x+y| r+|x-y| r)^{1 / r} \leqq 2^{1 / s^{\prime}}\left(|x|^{s}+|y|^{s}\right)^{1 / s}
$$

for all complex $x$ and $y$, with $1<s \leqq p \leqq r$, we deduce (1.4) (for $L^{p}$ ) from the inequalities [6, p. 148]

$$
\begin{aligned}
& \left\{\left(\int|x(t)+y(t)|^{p} d t\right)^{r / p}+\left(\int|x(t)-y(t)|^{p} d t\right)^{r / p}\right\}^{1 / r} \\
& \leqq\left\{\int\left(|x(t)+y(t)|^{r}+|x(t)-y(t)|^{r}\right)^{p / r} d t\right\}^{1 / p}, r \geqq p \\
& 2^{1 / s^{\prime}}\left\{\int\left(|x(t)|^{s}+|y(t)|^{s}\right)^{p / s} d t\right\}^{1 / p} \\
& \leqq 2^{1 / s^{\prime}}\left\{\left(\int|x(t)|^{p} d t\right)^{s / p}+\left(\int|y(t)| p d t\right)^{s / p}\right\}^{1 / s}, s \leqq p
\end{aligned}
$$

For, (2.1) shows that the left side of (2.3) is not less than the right side of (2.2). For $l^{p}$, we have the same inequalities with $\int$ replaced by $\sum[6$, p. 123$]$, and we draw the same conclusions.

3. Independent proof of (1.1). According to $\$ 2$, it is enough to prove

$$
\left(|x+y|^{p}+|x-y|^{p}\right)^{1 / p} \leqq 2^{1 / p^{\prime}}\left(|x|^{p}+|y|^{p}\right)^{1 / p}, \quad p \geqq 2 .
$$

\footnotetext{
4 See Bochner and Taylor [4] for a discussion of these spaces.
} 
We have, using first Jensen's inequality [6, p. 28] and then Hölder's inequality with index $p / 2[6, p .24]$,

$$
\begin{aligned}
\left(|x+y|^{p}+|x-y|^{p}\right)^{1 / p} & \leqq\left(|x+y|^{2}+|x-y|^{2}\right)^{1 / 2} \\
& =2^{1 / 2}\left(|x|^{2}+|y|^{2}\right)^{1 / 2} \\
& \leqq 2^{1 / 2}\left\{2^{(p-2) / p}\left(|x|^{p}+|y|^{p}\right)^{2 / p}\right\}^{1 / 2} \\
& =2^{1 / p^{\prime}}\left(|x|^{p}+|y|^{p}\right)^{1 / p}
\end{aligned}
$$

since $(p / 2)^{\prime}=p /(p-2)$.

This is the simplest proof of the uniform convexity of $L^{p}$ and $l^{p}$, $(p>2)$.

4. Proof of Clarkson's inequalities. We shall prove the following general theorem, of which Clarkson's inequalities are special cases: it asserts the truth of (1.2) and (1.3), respectively, when $p \geqq 2, r=p$, $s=p^{\prime}$, and when $1<p \leqq 2, r=p^{\prime}, s=p$.

THEOREM 1. If $x$ and $y$ are elements of $L^{p}$ or $l^{p},(p>1)$, then

$$
(\|x+y\| r+\|x-y\| r)^{1 / r} \leqq 2^{1 / s^{\prime}}\left(\|x\|^{s}+\|y\|^{s}\right)^{1 / s}
$$

whenever the point $(r, s)$ is in the common part of the regions of the $(r, s)$ plane determined by the inequalities

$$
r \geqq s \geqq r^{\prime}, \quad r \geqq p \geqq s>1 .
$$

As we saw in $\$ 2$, it is enough (since the second inequality (4.2) is satisfied) to establish

$$
\left(|x+y|^{r}+|x-y| r\right)^{1 / r} \leqq 2^{1 / s^{\prime}}\left(|x|^{s}+|y|^{s}\right)^{1 / s}
$$

for all complex $x$ and $y$. We now appeal to M. Riesz's convexity theorem, ${ }^{5}$ which, stated for the pair of linear forms $x+y$ and $x-y$, is that

$$
M_{a, b}=\log \max \frac{\left(|x+y|^{1 / a}+|x-y|^{1 / a}\right)^{a}}{\left(|x|^{1 / b}+|y|^{1 / b}\right)^{b}}
$$

is convex in the triangle $0 \leqq a \leqq b \leqq 1$.

Now, we have the identity

$$
\left(|x+y|^{2}+|x-y|^{2}\right)^{1 / 2}=2^{1 / 2}\left(|x|^{2}+|y|^{2}\right)^{1 / 2},
$$

and the trivial inequalities

$5[6$, p. 219]; a new and interesting proof (and generalization) has been given by Thorin [8].

${ }^{6}$ The maximum is taken over all $x$ and $y$. When $a$ or $b$ is zero, the numerator or denominator on the right is to be replaced by its limiting value as $a$ or $b$ approaches zero, namely $\max (|x+y|,|x-y|)$ or $\max (|x|,|y|)$. 


$$
\begin{aligned}
& \max (|x+y|,|x-y|) \leqq|x|+|y| \\
& \max (|x+y|,|x-y|) \leqq 2 \max (|x|,|y|) ;
\end{aligned}
$$

it follows easily that

$$
M_{1 / 2,1 / 2}=(1 / 2) \log 2, \quad M_{0,1}=0, \quad M_{0,0}=\log 2 .
$$

The graph of a continuous convex function lies below any chord. Since the linear function of $a$ and $b$ taking the values $\log 2,0$, $(1 / 2) \log 2$ at the points $(0,0),(0,1)$, and $(1 / 2,1 / 2)$ is $(1-b) \log 2$, we obtain from (4.4) (writing $a=1 / r, b=1 / s$ ), the inequality (4.3), valid for any $r$ and $s$ such that $(1 / r, 1 / s)$ is in the triangle whose vertices are $(0,0),(0,1)$, and $(1 / 2,1 / 2)$. These values of $r$ and $s$ are those satisfying $r>1$ and the first inequality (4.2).

The uniform convexity of $L^{p}$ and $l^{p}$ is a consequence of any case of Theorem 1; Clarkson's inequalities (1.2) and (1.3) are the strongest cases involving $p$ in a simple way. ${ }^{7}$ When $r=s=p \geqq 2$, we have the weakest inequality; we should therefore expect to be able to give the simplest proof in this case.

5. The uniform convexity of $\lambda^{p}\left\{\lambda^{q}\right\}$. It is convenient to have a shorthand notation for our $L^{p}$ and $l^{p}$ spaces. We shall use $\lambda$ to stand for either $L$ or $l$; a statement involving $\lambda$ is to be interpreted as the set of statements obtained by the reading of $L$ or $l$ for $\lambda$ in all possible ways.

The space $\lambda^{p}\{E\}$ is evidently uniformly convex if its elements satisfy any inequality of the form

$$
\left\{\mathfrak{M}_{p}^{r}[x+y]+\mathfrak{M}_{p}^{r}[x-y]\right\}^{1 / r} \leqq 2^{1 / s^{\prime}}\left\{\mathfrak{M}_{p}^{s}[x]+\mathfrak{M}_{p}^{8}[y]\right\}^{1 / s}
$$

with $r>1, s>1$.

The content of (5.1) will perhaps be clearer if we write it out explicitly for one of its cases. For $L^{p}\left\{l^{q}\right\}$, it states that for any sequences $\left\{x_{n}(t)\right\},\left\{y_{n}(t)\right\}$ of functions belonging to $L^{p}$,

$$
\begin{aligned}
& \left\{\left[\int\left(\sum\left|x_{n}(t)+y_{n}(t)\right|^{q}\right)^{p / q} d t\right]^{r / p}\right. \\
& \left.+\left[\int\left(\sum\left|x_{n}(t)-y_{n}(t)\right|^{q}\right)^{p / q} d t\right]^{r / p}\right\}^{1 / r} \\
& \leqq 2^{1 / s^{\prime}}\left\{\left[\int\left(\sum\left|x_{n}(t)\right|^{q}\right)^{p / q} d t\right]^{s / p}+\left[\int\left(\sum\left|y_{n}(t)\right|^{q}\right)^{p / q} d t\right]^{s / p}\right\}^{1 / s}
\end{aligned}
$$

7 The left side of (2.1) is an increasing function of $1 / r$; the right side is a decreasing function of $1 / s$. Hence we get a better inequality by increasing $1 / r$ or $1 / s$. 
provided that the right side is finite. For $l^{p}\left\{L^{q}\right\},(5.1)$ is the same inequality with $\int$ and $\sum$ interchanged.

Even when $E$ is $\lambda^{q}$, (5.1) is not true (naturally enough) for all choices of $r$ and $s$. Reasonable generalizations of Clarkson's inequalities are the cases of (5.1) described by the following table, when $E$ is $\lambda^{q}$.

\begin{tabular}{c|c|c|c}
$q$ & $p$ & $r$ & $s$ \\
\hline $1<q \leqq 2$ & $p \geqq q^{\prime}$ & $p$ & $p^{\prime}$ \\
$1<q \leqq 2$ & $q \leqq p \leqq q^{\prime}$ & $q^{\prime}$ & $q$ \\
$1<q \leqq 2$ & $1<p \leqq q$ & $p^{\prime}$ & $p$ \\
\hline$q \geqq 2$ & $p \geqq q$ & $p$ & $p^{\prime}$ \\
$q \geqq 2$ & $q^{\prime} \leqq p \leqq q$ & $q$ & $q^{\prime}$ \\
$q \geqq 2$ & $1<p \leqq q^{\prime}$ & $p^{\prime}$ & $p$
\end{tabular}

These inequalities are included in the following theorem.

THEOREM 2. If $x$ and $y$ are elements of $\lambda^{p}\left\{\lambda^{q}\right\},(p>1, q>1)$, then (5.1) is true whenever the point $(r, s)$ is in the common part of the regions determined by the inequalities

$$
r \geqq p \geqq s>1, \quad r(q-1) \geqq s \geqq r^{\prime}
$$

when $1<q \leqq 2$, or

$$
r \geqq p \geqq s>1, \quad r\left(q^{\prime}-1\right) \geqq s \geqq r^{\prime}
$$

when $q \geqq 2$.

As we have already seen, any case of Theorem 2 implies that the spaces $\lambda^{p}\left\{\lambda^{q}\right\}$ are uniformly convex for $p>1, q>1$.

To prove Theorem 2 , we observe that (5.1) is (1.4) with $\mathfrak{M}_{p}[\ldots]$ for $\|\cdots\|$. If we replace $|\cdots|$ by $\|\cdots\|$ in (2.2) and (2.3), the norm being the norm in $\lambda^{q},(2.2)$ and (2.3) are of course still valid, and we deduce that (5.1) holds if

$$
(\|x+y\| r+\|x-y\| r)^{1 / r} \leqq 2^{1 / s^{\prime}}\left(\|x\|^{s}+\|y\|^{s}\right)^{1 / s}
$$

with $1<s \leqq p \leqq r$, the norm being a $\lambda^{q}$ norm. We shall prove (5.4) in the cases specified by (5.2) and (5.3), and thus establish the corresponding cases of (5.1).

To do this, we generalize the argument of $\$ 4$; here Clarkson's inequalities will replace the equality

$$
\|x+y\|^{2}+\|x-y\|^{2}=2\left(\|x\|^{2}+\|y\|^{2}\right),
$$


which is valid only in euclidean spaces (Jordan and von Neumann [7]).

The form of Riesz's theorem used in $\$ 4$ is equivalent to another theorem of Riesz on bilinear forms; S. Bochner and the author [2] have observed that the latter remains true, with an appropriate interpretation, when the variables involved are elements of a Banach space. It follows that the convexity theorem for linear forms is also true (see \$6). For our special case it states that

$$
M_{a, b}=\log \sup \frac{\left(\|x+y\|^{1 / a}+\|x-y\|^{1 / a}\right)^{a}}{\left(\|x\|^{1 / b}+\|y\|^{1 / b}\right)^{b}}
$$

is convex in the triangle $0 \leqq a \leqq b \leqq 1$. (The least upper bound is taken as $x$ and $y$ vary over $\lambda^{q}$; the norms, of course, are $\lambda^{q}$ norms.)

We may now formulate (5.4) as

$$
M_{1 / r, 1 / 8} \leqq\left(1 / s^{\prime}\right) \log 2, \quad 1<s \leqq p \leqq r<\infty .
$$

We have, in $\lambda^{q}$ (and in fact in any Banach space),

$$
M_{0,0}=\log 2, \quad M_{0,1}=0 .
$$

Clarkson's inequalities (1.2) and (1.3) give us

$$
M_{1 / q, 1 / q^{\prime}} \leqq(1 / q) \log 2, q \geqq 2 ; \quad M_{1 / q^{\prime}, 1 / q} \leqq\left(1 / q^{\prime}\right) \log 2,1<q \leqq 2 .
$$

Consequently (5.4) is true whenever $(1 / r, 1 / s)$ is in the triangle with vertices $(0,0),(0,1),\left(1 / q, 1 / q^{\prime}\right)$ if $q \geqq 2$, or in the triangle with vertices $(0,0),(0,1),\left(1 / q^{\prime}, 1 / q\right)$ if $1<q \leqq 2$. These points correspond to points $(r, s)$, with $r>1$, satisfying the second inequalities (5.2) or (5.3). This completes the proof of Theorem 2 .

It should be mentioned that certain cases of (5.2), adequate to establish the uniform convexity of $\lambda^{p}\left\{\lambda^{q}\right\}$ for some (but not all) $p$ and $q$, follow directly from Clarkson's inequalities without any convexity theorem. For example, if $q \geqq 2$, (5.4) becomes (1.2) for $r=q$, $s=q^{\prime}$; hence (5.4) still holds for $r \geqq q, s \geqq q^{\prime}$; therefore, if $p \geqq q^{\prime}$, we can take $r=s=p$ and thus establish the uniform convexity of $\lambda^{p}\left\{\lambda^{q}\right\}$ for $p \geqq q^{\prime}, q \geqq 2$.

6. The abstract convexity theorem. Let $E$ be a Banach space, with elements $x$; let its conjugate space have elements $f$ (linear functionals on $E$ ). Let $N_{c, b}$ be the least upper bound of

$$
\left|\sum_{i=1}^{m} \sum_{j=1}^{n} a_{i j} f_{j}\left(x_{i}\right)\right|
$$

for 


$$
\sum_{i=1}^{m}\left\|x_{i}\right\|^{1 / b} \leqq 1, \quad \sum_{j=1}^{n}\left\|f_{j}\right\|^{1 / c} \leqq 1 .
$$

It is known ${ }^{8}$ that $\log N_{c, b}$ is convex in the triangle $0 \leqq b \leqq 1,0 \leqq c \leqq 1$, $b+c \geqq 1$.

We wish to show that if $M_{a, b}$ is the least upper bound of the expression

$$
\left\{\sum_{j=1}^{n}\left\|\sum_{i=1}^{m} a_{i j} x_{i}\right\|^{1 / a}\right\}^{a}
$$

for

$$
\sum_{i=1}^{m}\left\|x_{i}\right\|^{1 / b} \leqq 1
$$

then $\log M_{a, b}$ is convex in the triangle $0 \leqq a \leqq b \leqq 1$. This we do by showing that $M_{a, b}=N_{c, b}$ if $c=1-a$.

Let $\left\{f_{j}\right\}$ and $\left\{x_{i}\right\}$ be arbitrary sets satisfying (6.2) with $c=1-a$. Then, by Hölder's inequality,

$$
\begin{aligned}
\left|\sum_{i=1}^{m} \sum_{j=1}^{n} a_{i j} f_{j}\left(x_{i}\right)\right| & =\left|\sum_{j=1}^{n} f_{j}\left(\sum_{i=1}^{m} a_{i j} x_{i}\right)\right| \\
& \leqq\left(\sum_{j=1}^{n}\left\|f_{j}\right\|^{1 /(1-a)}\right)^{1-a}\left(\sum_{j=1}^{n}\left\|\sum_{i=1}^{m} a_{i j} x_{i}\right\|^{1 / a}\right)^{a} \\
& \leqq M_{a, b} .
\end{aligned}
$$

Consequently ${ }^{9}$

$$
N_{c, b} \leqq M_{a, b} .
$$

Now let $\left\{x_{i}\right\}$ be an arbitrary set satisfying (6.4). We can find ${ }^{10}$ linear functionals $f_{i},(j=1,2, \cdots, n)$, such that

$$
\begin{aligned}
f_{j}\left(\sum_{i=1}^{m} a_{i j} x_{i}\right) & =\left\|\sum_{i=1}^{m} a_{i j} x_{i}\right\| \cdot\left\|f_{j}\right\|, & & j=1,2, \cdots, n ; \\
\left\|f_{j}\right\|^{1 / c} & =A\left\|\sum_{i=1}^{m} a_{i j} x_{i}\right\|^{1 / a}, & j & =1,2, \cdots, n,
\end{aligned}
$$

with constant $A \neq 0$; and $\sum_{j=1}^{n}\left\|f_{j}\right\| 1 / c=1$. For these $f_{j}$ we have, using the conditions for equality in Hölder's inequality,

${ }^{8}$ Boas and Bochner [2, p. 64].

${ }^{9}$ This simple argument was suggested by the referee.

${ }^{10}$ Banach $[1$, p. 55]. 


$$
\begin{aligned}
\sum_{j=1}^{n} \sum_{i=1}^{m} a_{i j} f_{j}\left(x_{i}\right) & =\sum_{j=1}^{n} f_{j}\left(\sum_{i=1}^{m} a_{i j} x_{i}\right) \\
& =\sum_{j=1}^{n}\left\|f_{j}\right\| \cdot\left\|\sum_{i=1}^{m} a_{i j} x_{i}\right\| \\
& =\left(\sum_{j=1}^{n}\left\|f_{j}\right\| \|^{1 /(1-a)}\right)^{1-a}\left(\sum_{j=1}^{n}\left\|\sum_{i=1}^{m} a_{i j} x_{i}\right\|^{1 / a}\right)^{a} \\
& =\left(\sum_{j=1}^{n}\left\|\sum_{i=1}^{m} a_{i j} x_{i}\right\|^{1 / a}\right)^{a} .
\end{aligned}
$$

Hence $M_{a, b} \leqq N_{c, b}$ if $c=1-a$; this, with (6.5), completes the proof.

\section{REFERENCES}

1. S. Banach, Théorie des Opérations Linéaires, 1932.

2. R. P. Boas, Jr., and S. Bochner, On a theorem of M. Riesz for Fourier series, Journal of the London Mathematical Society, vol. 14 (1939), pp. 62-73.

3. S. Bochner, Integration von Funktionen, deren Werte die Elemente eines Vektorraumes sind, Fundamenta Mathematicae, vol. 20 (1933), pp. 262-276.

4. S. Bochner and A. E. Taylor, Linear functionals on certain spaces of abstractlyvalued functions, Annals of Mathematics, (2), vol. 39 (1938), pp. 913-944.

5. J. A. Clarkson, Uniformly convex spaces, Transactions of this Society, vol. 40 (1936), pp. 396-414.

6. G. H. Hardy, J. E. Littlewood, and G. Polya, Inequalities, 1934.

7. P. Jordan and J. von Neumann, On inner products in linear, metric spaces, Annals of Mathematics, (2), vol. 36 (1935), pp. 719-723.

8. G. O. Thorin, An extension of a convexity theorem due to M. Riesz, Fysiografiska Sällskapets i Lund, Förhandlingar (Proceedings of the Royal Physiographic Society at Lund), vol. 8 (1938), no. 14.

Cambridge, England 